FERMILAB-Pub-93/399-A

SUSSEX-AST $94 / 2-1$

astro-ph/9402021

(February 1994)

submitted to Physical Review D

\title{
SECOND-ORDER RECONSTRUCTION OF THE INFLATIONARY POTENTIAL
}

\author{
Andrew R. Liddle ${ }^{1}$ and Michael S. Turner ${ }^{2,3}$ \\ ${ }^{1}$ Astronomy Centre, Division of Physics and Astronomy \\ University of Sussex, Brighton BN1 9QH, UK \\ ${ }^{2}$ NASA/Fermilab Astrophysics Center \\ Fermi National Accelerator Laboratory, Batavia, IL 60510-0500 \\ ${ }^{3}$ Departments of Physics and of Astronomy \& Astrophysics \\ Enrico Fermi Institute, The University of Chicago, Chicago, IL 60637-1433
}

\begin{abstract}
To first order in the deviation from scale invariance the inflationary potential and its first two derivatives can be expressed in terms of the spectral indices of the scalar and tensor perturbations, $n$ and $n_{T}$, and their contributions to the variance of the quadrupole CBR temperature anisotropy, $S$ and $T$. In addition, there is a "consistency relation" between these quantities: $n_{T}=-\frac{1}{7} \frac{T}{S}$. We discuss the overall strategy of perturbative reconstruction and derive the second-order expressions for the inflationary potential and its first two derivatives and the first-order expression for its third derivative, all in terms of $n, n_{T}, S, T$, and $d n / d \ln k$. We also obtain the second-order consistency relation, $n_{T}=-\frac{1}{7} \frac{T}{S}\left[1+0.11 \frac{T}{S}+0.15(n-1)\right]$. As an example we consider the exponential potential, the only known case where exact analytic solutions for the perturbation spectra exist. We reconstruct the potential via Taylor expansion (with coefficients calculated at both first and second order), and introduce the Padé approximant as a greatly improved alternative.
\end{abstract}

PACS number(s): 98.80.Cq, 98.70.Vc 


\section{Introduction}

In inflationary models quantum fluctuations excited on very small length scales $\left(\sim H^{-1} \sim\right.$ $\left.10^{-23} \mathrm{~cm}\right)$ are stretched to astrophysical scales $\left(\sim 10^{25} \mathrm{~cm}\right)$ by the tremendous growth of the scale factor during inflation ( $H$ is the value of Hubble parameter during inflation) [1]. This results in almost scale-invariant spectra of scalar (density) [2] and tensor (gravitationalwave) [3 metric perturbations. Together with the prediction of a spatially-flat Universe they provide the means for testing the inflationary paradigm. The tensor fluctuations lead to cosmic background radiation (CBR) anisotropy and a stochastic background of gravitational waves with wavelengths from about $1 \mathrm{~km}$ to over $10^{4} \mathrm{Mpc}$. The scalar fluctuations also lead to CBR anisotropy and seed the formation of structure in the Universe.

The amplitudes and spectral indices of the metric fluctuations can be expressed in terms of the inflationary potential and its derivatives, evaluated at the value of the scalar field when astrophysically interesting scales crossed outside the horizon during inflation (from galactic scales to the presently observable Universe, corresponding to the eight e-foldings about 50 e-folds or so before the end of inflation). Techniques have been developed for relating the scalar and tensor spectra to the potential and its derivatives in an expansion whose small parameter is the deviation from scale invariance 44, 5]. In particular, the spectral indices and the power spectra of the fluctuations today can be written as [5]

$$
\begin{gathered}
n=1-\frac{x_{50}^{2}}{8 \pi}+\frac{m_{\mathrm{Pl}} x_{50}^{\prime}}{4 \pi}, \quad n_{T}=-\frac{x_{50}^{2}}{8 \pi}, \\
P(k)=A k^{n}|T(k)|^{2}, \quad P_{T}(k)=A_{T} k^{n_{T}-3}\left|T_{T}(k)\right|^{2}\left(\frac{3 j_{1}\left(k \tau_{0}\right)}{k \tau_{0}}\right)^{2}, \\
A=\frac{1024 \pi^{3} k_{50}^{1-n}}{75 H_{0}^{4}}\left[1+\frac{7}{6} n_{T}+\left(-\frac{7}{3}+\ln 2+\gamma\right)(n-1)\right] \frac{V_{50}}{m_{\mathrm{Pl}}^{4} x_{50}^{2}}, \\
A_{T}=\frac{8 k_{50}^{-n_{T}}}{3 \pi}\left[1+\left(-\frac{7}{6}+\ln 2+\gamma\right) n_{T}\right] \frac{V_{50}}{m_{\mathrm{Pl}}^{4}} .
\end{gathered}
$$

Here $k$ is the comoving wavenumber, $x=m_{\mathrm{Pl}} V^{\prime} / V$ measures the steepness of the potential, prime denotes derivative with respect to the scalar field that drives inflation, subscript 50 indicates that the quantity is to be evaluated 50 e-folds before the end of inflation, [ $m_{\mathrm{Pl}}=$

\footnotetext{
${ }^{1}$ Several minor errors in Ref. [5] have been corrected here: the factor of $H_{0}^{3+n}$ in Eq. (A5) should be $H_{0}^{4}$; the factor of $H_{0}^{3+n}$ in Eq. (A7) should be $2^{n-1} H_{0}^{4}$; the factor of 1.1(n-1) in Eq. (A8) is more precisely $1.3(n-1)$; the factor of $1.2 n_{T}$ in Eq. (A14) is more precisely $1.4 n_{T}$.

${ }^{2}$ The point about which the potential is expanded is in principle arbitrary. However, the spectral indices $n$ and $n_{T}$ can only plausibly be measured on scales from $1 \mathrm{Mpc}-10^{4} \mathrm{Mpc}$ and $S$ and $T$ depend upon perturbations on these same scales, so it makes sense to choose the expansion point to correspond to when these scales crossed outside the horizon during inflation; in addition, by taking $k_{50} \tau_{0}=1$ several expressions simplify. The precise number of e-folds before the end of inflation when these scales crossed outside the horizon depends logarithmically upon the energy scale of inflation and the reheat temperature, see Refs. 化, 5. 60; for the sake of definiteness we take this number to be 50, which can easily be changed to the correct value for a given inflationary model.
} 
$1.22 \times 10^{19} \mathrm{GeV}$ is the Planck mass, $H_{0}$ is the present value of the Hubble constant, $\tau_{0} \simeq 2 H_{0}^{-1}$ is the present conformal age of the Universe, and $\gamma \simeq 0.577$ is Euler's constant. Scaleinvariant metric perturbations correspond to $(n-1)=n_{T}=0$. The functions $T(k)$ and $T_{T}(k)$ are the transfer functions for scalar [7] and tensor [8] metric perturbations respectively; for $k \tau_{0} \ll 100$, both $T(k)$ and $T_{T}(k) \rightarrow 1$. The expressions for $n$ and $n_{T}$ are given to lowest order in the deviation from scale invariance (hereafter, referred to as first order), and the expressions for $A$ and $A_{T}$ include the lowest-order term as well as the next correction (hereafter, referred to second order) [9].

From these expressions the consequences of the scalar and tensor metric fluctuations may be computed. In particular, the contributions to the variance of the angular power spectrum of the CBR anisotropy on large angular scales $(l \ll 200)$ which arise predominantly due to the Sachs-Wolfe effect are given by [8]

$$
\begin{aligned}
\left\langle\left|a_{l m}^{S}\right|^{2}\right\rangle & =\frac{H_{0}^{4}}{2 \pi} \int_{0}^{\infty} k^{-2} P(k)\left|j_{l}\left(k \tau_{0}\right)\right|^{2} d k \\
\left\langle\left|a_{l m}^{T}\right|^{2}\right\rangle & =36 \pi^{2} \frac{\Gamma(l+3)}{\Gamma(l-1)} A_{T} \int_{0}^{\infty} k^{n_{T}+1}\left|F_{l}(k)\right|^{2}\left|T_{T}(k)\right|^{2} d k \\
F_{l}(k) & =-\int_{\tau_{\mathrm{LSS}}}^{\tau_{0}} \frac{j_{2}\left(k \tau_{0}\right)}{k \tau_{0}}\left(\frac{j_{l}\left(k \tau_{0}-k \tau\right)}{\left(k \tau_{0}-k \tau\right)^{2}}\right) d \tau
\end{aligned}
$$

where $\tau_{\mathrm{LSS}} \simeq \tau_{0} /\left(1+z_{\mathrm{LSS}}\right)^{1 / 2} \approx \tau_{0} / 35$ is the conformal age at last scattering $\left(z_{\mathrm{LSS}} \simeq 1100\right)$ and $j_{l}$ is the spherical Bessel function of order $l$. (We note in passing that both expressions are based upon the approximation that the Universe is matter-dominated at last-scattering; the small contribution of radiation, about 10\%-20\%, leads to corrections [8] that would have to be included in a more accurate treatment. The corrections to the quadrupole anisotropy are small.)

The contribution of scalar and tensor metric perturbations to the observer averaged variance of the quadrupole CBR anisotropy can be computed numerically [5]

$$
\begin{aligned}
& S \equiv \frac{5\left\langle\left|a_{2 m}^{S}\right|^{2}\right\rangle}{4 \pi} \simeq 2.2\left[1+1.2 n_{T}+0.08(n-1)\right] \frac{V_{50}\left(k_{50} \tau_{0}\right)^{1-n}}{m_{\mathrm{Pl}}^{4} x_{50}^{2}} \\
& T \equiv \frac{5\left\langle\left|a_{2 m}^{T}\right|^{2}\right\rangle}{4 \pi} \simeq 0.61\left[1+1.4 n_{T}\right] \frac{V_{50}\left(k_{50} \tau_{0}\right)^{-n_{T}}}{m_{\mathrm{Pl}}^{4}}
\end{aligned}
$$

where the dependence upon $(n-1)$ and $n_{T}$ is given to first-order. In evaluating these expressions the effect of transfer functions is negligible as the integrals are dominated by $k \tau_{0} \sim 2$. For simplicity, following footnote 2 we henceforth omit factors of $\left(k_{50} \tau_{0}\right)^{1-n}$ and $\left(k_{50} \tau_{0}\right)^{-n_{T}}$; they are easily re-inserted if needed. 


\subsection{First-order reconstruction}

We choose $S, T, n_{T}$, and $(n-1)$ as a convenient set of observables; other choices are possible and can be easily transformed to our set. Since $S, T, n$, and $n_{T}$ are expressed in terms of the potential and its first two derivatives, one can invert the expressions to solve for the potential and its first two derivatives in terms of $S, T, n$, and $n_{T}$ plus a "consistency relation." Those expressions are [12]

$$
\begin{aligned}
V_{50} / m_{\mathrm{Pl}}^{4} & =1.65\left(1-1.4 n_{T}\right) T, \\
& =1.65\left(1+0.20 \frac{T}{S}\right) T, \\
V_{50}^{\prime} / m_{\mathrm{Pl}}^{3} & = \pm 8.3 \sqrt{-n_{T}} T, \\
& = \pm 8.3 \sqrt{\frac{1}{7} \frac{T}{S}} T, \\
V_{50}^{\prime \prime} / m_{\mathrm{Pl}}^{2} & =21\left[(n-1)-3 n_{T}\right] T, \\
& =21\left[(n-1)+0.43 \frac{T}{S}\right] T, \\
n_{T} & =-\frac{1}{7} \frac{T}{S} .
\end{aligned}
$$

In the second expressions for the potential and its first two derivatives we have used the consistency relation to express $n_{T}$ in terms of $\frac{T}{S}$, as $\frac{T}{S}$ should be easier to measure [10]. Note that the sign of $V^{\prime}$ cannot be determined as it can be changed by a field redefinition $\phi \rightarrow-\phi$, though a specific choice here determines the signs of various later expressions. This procedure actually generates the full second-order term for $V_{50}$, while the other expressions are first-order.

In order to actually reconstruct the inflationary potential over the eight or so e-folds relevant for astrophysics from its value and first two derivatives one needs to relate the number of e-foldings from the end of inflation, $N$ (where $d N / d t=-H$ ), to the value of the scalar field. To lowest order the equation for $d \phi / d N$ follows from the slow-roll equation for the evolution of $\phi$ and is given by

$$
\frac{d \phi}{d N} \simeq \frac{m_{\mathrm{Pl}}^{2}}{8 \pi} \frac{V^{\prime}}{V},
$$

where to lowest order the right-hand side is just $\sqrt{-n_{T}} m_{\mathrm{Pl}} / \sqrt{8 \pi}$.

In the next section we discuss the overall strategy of perturbative reconstruction, and in the following section go on to derive the second-order expressions for the potential and its first two derivatives, for the equation relating $\phi$ and $N$, and for the consistency relation, as well as the first-order expression for the third derivative. We finish with a brief discussion of our results.

\footnotetext{
${ }^{3}$ For example, in separating the tensor and scalar contributions to CBR anisotropy one might measure $l(l+1)\left\langle\left|a_{l m}\right|^{2}\right\rangle$ for four values of $l$ (or ranges centered on four different values of $l$ ); see e.g., Refs. 10, 11. From these measurements and the known dependence of $l(l+1)\left\langle\left|a_{l m}^{S}\right|^{2}\right\rangle / 4 \pi \approx S(l / 2)^{n-1}$ and $l(l+1)\left\langle\left|a_{l m}^{T}\right|^{2}\right\rangle / 4 \pi \approx$ $T(l / 2)^{n_{T}}$ upon $S,(n-1), T$, and $n_{T}$, cf. Eqs. (5) and (6), our chosen observables can be extracted.
} 


\section{Perturbative Reconstruction Strategy}

While one can hope to learn about the potential over the interval that affects astrophysical scales, it is probably not realistic to hope to learn much about the potential globally without some additional a priori knowledge (e.g., the functional form of the potential). Th The fundamental goal of perturbative reconstruction is to use a finite set of data to reconstruct the inflationary potential over the interval where the eight or so e-foldings of inflation relevant to astrophysics took place. The observational data all trace to the scalar and tensor metric perturbations, whose observable consequences can be expressed in terms of the inflationary potential $V$ and its derivatives $V^{(m)}$ evaluated at some convenient point in this interval. (For brevity, in this section we drop the subscript '50' that indicates where the potential and its derivatives are to be evaluated.) Once the observables, e.g., $n_{T},(n-1), S, T$ and so on, have been expressed in terms of the potential and its derivatives, these expressions can be inverted to express the potential and its derivatives in terms of the observables, as well as a consistency relation. From these the potential can be recovered by expansion.

In principle, the observables depend upon all the derivatives of the potential, making the problem appear intractable. If one is willing to restrict the problem to flat potentials which lead to nearly scale-invariant perturbations and nearly exponential inflation, the problem can be made manageable. (In the scale-invariant limit the potential is precisely constant and all its derivatives vanish.) In the nearly scale-invariant limit we have a set, albeit infinite, of small parameters to expand in: $m_{\mathrm{Pl}}^{m} V^{(m)} / V$; as we shall describe, when calculating to a given accuracy only a small number of derivatives are needed. Put another way, the terms involving more derivatives or higher derivatives are of higher order. To be more specific, to lowest order

$$
\begin{aligned}
T & \sim \mathcal{O}\left(V / m_{\mathrm{Pl}}^{4}\right) \\
n_{T}, T / S & \sim \mathcal{O}\left[\left(m_{\mathrm{Pl}} V^{\prime} / V\right)^{2}\right], \\
(n-1) & \sim \mathcal{O}\left[\left(m_{\mathrm{Pl}} V^{\prime} / V\right)^{2}\right]+\mathcal{O}\left[m_{\mathrm{Pl}}^{2} V^{\prime \prime} / V\right]
\end{aligned}
$$

that is, only the potential and its first two derivatives come into play.

Corrections from higher derivatives come into play because of the variation of the potential and its first two derivatives during the Hubble time or so that a given scale is crossing outside the horizon and is becoming a classical metric perturbation. It is straightforward to write down the form of the higher derivative terms expected by using the fact that the variation of a given derivative over a Hubble due to a higher derivative is:

$$
\delta V^{(n)} \sim V^{(m)} \delta \phi^{m-n} \sim\left(m_{\mathrm{Pl}}^{2} V^{\prime} / V\right)^{m-n} V^{(m)},
$$

where the final expression follows by using $d \phi / d N \sim m_{\mathrm{Pl}}^{2} V^{\prime} / V$. The form of the higher-order

\footnotetext{
${ }^{4}$ The one possible exception involves the accurate measurement of the stochastic background of gravitational waves on scales from $1 \mathrm{~km}$ to $3000 \mathrm{Mpc}$ (corresponding to $N \simeq 0-50$ ) in which case the inflationary potential could be mapped out directly since the amplitude of the tensor perturbation on a given scale is related to the value of the potential.
} 
terms in the expansions of $T, n_{T}, T / S$, and $n$ can now be written down directly:

$$
\begin{aligned}
T & \sim \mathcal{O}\left(V / m_{\mathrm{Pl}}^{4}\right)\left[1+\mathcal{O}\left[\left(m_{\mathrm{Pl}} V^{\prime} / V\right)^{2}\right]+\mathcal{O}\left[\left(m_{\mathrm{Pl}}^{2} V^{\prime \prime} / V\right)\left(m_{\mathrm{Pl}} V^{\prime} / V\right)^{2}\right]+\cdots\right] \\
n_{T}, T / S & \sim \mathcal{O}\left[\left(m_{\mathrm{Pl}} V^{\prime} / V\right)^{2}\right]+\mathcal{O}\left[\left(m_{\mathrm{Pl}}^{2} V^{\prime \prime} / V\right)\left(m_{\mathrm{Pl}} V^{\prime} / V\right)^{2}\right] \\
& +\mathcal{O}\left[\left(m_{\mathrm{Pl}}^{3} V^{(3)} / V\right)\left(m_{\mathrm{Pl}} V^{\prime} / V\right)^{3}\right]+\cdots \\
n-1 & \sim \mathcal{O}\left[\left(m_{\mathrm{Pl}} V^{\prime} / V\right)^{2}\right]+\mathcal{O}\left[m_{\mathrm{Pl}}^{2} V^{\prime \prime} / V\right]+\mathcal{O}\left[\left(m_{\mathrm{Pl}}^{3} V^{(3)} / V\right)\left(m_{\mathrm{Pl}} V^{\prime} / V\right)\right] \\
& +\mathcal{O}\left[\left(m_{\mathrm{Pl}}^{3} V^{(3)} / V\right)\left(m_{\mathrm{Pl}}^{2} V^{\prime \prime} / V\right)\left(m_{\mathrm{Pl}} V^{\prime} / V\right)\right]+\mathcal{O}\left[\left(m_{\mathrm{Pl}}^{3} V^{(3)} / V\right)\left(m_{\mathrm{Pl}} V^{\prime} / V\right)^{3}\right]+\cdot
\end{aligned}
$$

The expansion for $V$ begins with a term that involves no derivatives; the next term involves two derivatives; the next four derivatives, and so on. The expansions for $n_{T}, T / S$, and $(n-1)$ begin with terms involving two derivatives; followed by terms involving four derivatives; and so on. In the previous literature, the lowest-order term has been referred to as first-order; the next term, which involves two additional derivatives, has been referred to as second-order; and so on. Explicit expressions for the second-order terms are given in Refs. 9, 13]; some of the third-order terms for $(n-1)$ are given in Ref. [14].

In the next three subsections we address the convergence of the Taylor series for the potential and the relative sizes of the terms in the expansions for the observables. We show that for a very general class of potentials that lend themselves to reconstruction that the higher-order terms in these expansions are smaller and are bounded by $n_{T} / \Delta N^{m / 2-1}$, where $m$ is the number of derivatives in the term, and further, that the Taylor series for the potential is absolutely convergent.

Before going on, let us remind the reader of a very useful fact and mention some notation. The variation in the scalar field over the eight relevant e-folds of inflation will be needed in many places; it is $\Delta \phi / m_{\mathrm{Pl}} \sim\left(m_{\mathrm{Pl}} V^{\prime} / V\right) \Delta N$, where $\Delta N \sim 8$ and throughout we use $\Delta$ to indicate the change in a quantity over the eight relevant e-folds. Since $n_{T} \sim\left(m_{\mathrm{Pl}} V^{\prime} / V\right)^{2}$, we will use $n_{T}^{1 / 2}$ to characterize the size of $m_{\mathrm{Pl}} V^{\prime} / V$. While it is actually $(n-1)-3 n_{T}$ and not $(n-1)$ whose lowest order term is given by $m_{\mathrm{Pl}}^{2} V^{\prime \prime} / V$, for simplicity we will often use $(n-1)$ to characterize the size of $m_{\mathrm{Pl}}^{2} V^{\prime \prime} / V$.

\subsection{Scale-free potentials}

Let us begin with a very simple class of potentials before we consider the general case. These are potentials without a scale other than an overall normalization; e.g., $V(\phi)=V_{0} \exp (-\beta \phi)$, $V(\phi)=a \phi^{b}$, or $V(\phi)=a \phi^{-b}$. For such potentials there is but a single expansion parameter since

$$
\frac{m_{\mathrm{Pl}}^{m} V^{(m)}}{V} \sim \mathcal{O}\left[\left(m_{\mathrm{Pl}} V^{\prime} / V\right)^{m}\right]
$$

For the potentials given above, Eq. (22) follows directly; in the absence of a more quantitative definition of scale-free we shall use Eq. (22) as the definition.

If we use $n_{T} \sim\left(m_{\mathrm{Pl}} V^{\prime} / V\right)^{2}$ to characterize the deviation from scale-invariance, it follows that

$$
\frac{m_{\mathrm{Pl}}^{m} V^{(m)}}{V} \sim \mathcal{O}\left[n_{T}^{m / 2}\right]
$$


with higher-order corrections to the expression for $m_{\mathrm{Pl}}^{m} V^{(m)} / V$ increasing as powers of $n_{T}$. For such potentials $n-1$ and $n_{T}$ are necessarily of the same order, which is not true in the general case. The convergence of the power series for $V(\phi)$ over the interval of $\Delta N \sim 8$ e-folds is manifest, as the contribution of the $m$-th derivative to the Taylor expansion is

$$
\frac{\Delta V_{m}}{V} \sim \frac{1}{m !} \frac{V^{(m)} \Delta \phi^{m}}{V} \sim \frac{\left(n_{T} \Delta N\right)^{m}}{m !} .
$$

For scale-free potentials Eq. (22) provides the ordering of terms in the expansion of the observables in terms of the derivatives of the potential very directly: the order of a term involving $m$ derivatives is $\left(m_{\mathrm{Pl}} V^{\prime} / V\right)^{m} \sim \mathcal{O}\left(n_{T}^{m / 2}\right)$. For example, in expression Eq. (21) for $(n-1)$ the first two terms are of the order of $n_{T}$; the next is of the order of $n_{T}^{2}$; and the final two are of the order of $n_{T}^{3}$.

\subsection{Strong reconstructability}

A priori we do not know the form of the potential and thus whether or not it is scale-free; therefore, it is important to address the most generic case possible. Lacking a priori knowledge of the potential, one can take advantage of the observational data itself for guidance in reconstruction. In the near term the observational data available are likely to be a handful of numbers, e.g., $n_{T},(n-1), S$, and $T$. A reasonable, robust, and pragmatic criterion for reconstructability is that the spectral indices do not vary greatly over the eight e-folds of interest; that is,

$$
\frac{\left|\Delta n_{T}\right|}{\left|n_{T}\right|}<\delta, \quad \frac{|\Delta(n-1)|}{|(n-1)|}<\delta,
$$

where $\delta$ is some suitably small number. We shall refer to this as "strong reconstructability," or SR.

Since the scalar and tensor spectral indices depend upon the first two derivatives of the inflationary potential, SR can be quantified in the following way: $V^{\prime}$ and $V^{\prime \prime}$ should not vary significantly over the interval of inflation affecting astrophysically interesting scales. This in turn constrains the higher derivatives of the potential through their contributions to the Taylor expansions of $V^{\prime}$ and $V^{\prime \prime}$ :

$$
\begin{aligned}
\frac{\Delta V^{\prime}}{V^{\prime}}<\delta \Rightarrow \frac{V^{(m)} \Delta \phi^{m-1}}{(m-1) ! V}<\delta \frac{V^{\prime}}{V} & \text { for } m \geq 2 \\
\frac{\Delta V^{\prime \prime}}{V^{\prime \prime}}<\delta & \Rightarrow \frac{V^{(m)} \Delta \phi^{m-2}}{(m-2) ! V}<\delta \frac{V^{\prime \prime}}{V} \quad \text { for } m \geq 3
\end{aligned}
$$

Again using $\Delta \phi / m_{\mathrm{Pl}} \sim\left(m_{\mathrm{Pl}} V^{\prime} / V\right) \Delta N$, these bounds become

$$
\begin{aligned}
\frac{m_{\mathrm{Pl}}^{m} V^{(m)}}{V} & <\left(\frac{m_{\mathrm{Pl}} V^{\prime}}{V}\right)^{-m+2} \Delta N^{-m+1} \delta(m-1) ! \\
& <\mathcal{O}\left[n_{T}^{-m / 2+1} \Delta N^{-m+1} \delta(m-1) !\right] \quad \text { for } m \geq 2
\end{aligned}
$$




$$
\begin{aligned}
\frac{m_{\mathrm{Pl}}^{m} V^{(m)}}{V} & <\left(\frac{m_{\mathrm{Pl}} V^{\prime}}{V}\right)^{-m+2}\left(\frac{m_{\mathrm{Pl}}^{2} V^{\prime \prime}}{V}\right) \Delta N^{-m+2} \delta(m-2) ! \\
& <\mathcal{O}\left[(n-1) n_{T}^{-m / 2+1} \Delta N^{-m+2} \delta(m-2) !\right] \quad \text { for } m \geq 3,
\end{aligned}
$$

where in the final expressions we have used the fact that $n_{T} \sim \mathcal{O}\left[\left(m_{\mathrm{Pl}} V^{\prime} / V\right)^{2}\right]$ and that $(n-1) \sim \mathcal{O}\left[m_{\mathrm{Pl}}^{2} V^{\prime \prime} / V\right]$.

These constraints to the derivatives of the potential are weaker than the ones we derived for scale-free potentials, but are more generally applicable and serve the same purpose. The second of these implies that the Taylor expansion for the potential is absolutely convergent, as it bounds the contribution to $V(\phi)$ from the $m$-th derivative

$$
\frac{\Delta V_{m}}{V} \sim \frac{V^{(m)} \Delta \phi^{m}}{m ! V}<\mathcal{O}\left(\frac{n_{T}(n-1) \Delta N^{2} \delta}{m(m-1)}\right) .
$$

Constraints (28) and (29) also serve to order terms in the expansions of the observables in terms of the potential and its derivatives. For example, Eq. (21) for the scalar index includes terms of order $\left(m_{\mathrm{Pl}} V^{\prime} / V\right)^{2}, m_{\mathrm{Pl}} V^{\prime \prime} / V,\left(m_{\mathrm{Pl}} V^{\prime} / V\right)\left(m_{\mathrm{Pl}}^{3} V^{(3)} / V\right)$. Our SR bounds tell us nothing about the relative sizes of first and second terms (the first-order terms), though they imply that the second term must be smaller than $\delta / \Delta N$. Based on the SR bounds, the third term (second-order term) must be less than both $\delta / \Delta N^{2}$ and $\left(m_{\mathrm{Pl}}^{2} V^{\prime \prime} / V\right) \delta / \Delta N$, and so it is necessarily of higher order than the second term. The higher-derivative terms, $\left(m_{\mathrm{Pl}} V^{\prime} / V\right)^{3}\left(m_{\mathrm{Pl}}^{3} V^{(3)} / V\right)$ and $\left(m_{\mathrm{Pl}} V^{\prime} / V\right)\left(m_{\mathrm{Pl}}^{2} V^{\prime \prime} / V\right)\left(m_{\mathrm{Pl}}^{3} V^{(3)} / V\right)$ terms are of even higher order: the former must be less than $\delta / \Delta N^{2}$ times the first term in the expansion for $(n-1)$ and less than $n_{T} \delta / \Delta N$ times the second term in the expansion for $(n-1)$, while the latter must be less than $\delta / \Delta N^{2}$ times the second term in the expansion for $(n-1)$. The ordering of the terms in the derivative expansion for $(n-1)$ is clear: more derivatives are suppressed by powers of $\Delta N$. In particular, a term involving $m$ derivatives can be no larger than $n_{T} \delta / \Delta N^{m / 2-1}$.

Before ending this subsection, we mention an interesting possibility: for models with very large deviations from scale-invariance the data may some day be good enough that a small fractional change in the spectral index is observable. A case in point is intermediate inflation [15], where the scalar index may be greater than unity and may decrease significantly. In particular, the potential for intermediate inflation is scale-free and $d n / d \ln k \sim(n-1)^{2}$ so that $\Delta(n-1) /(n-1) \sim \Delta N(n-1)$. The SR bounds still apply, and additionally, the new observable $d n / d \ln k$ allows one to determine $V^{\prime \prime \prime}$ at lowest order (as described in section 3 ).

\subsection{Weak reconstructability}

The pragmatic criteria of SR discussed above can be relaxed somewhat, without sacrificing the convergence of the Taylor series for the potential or the ordering of terms in the expansions for the observables. Suppose that one, or even both, of the spectral indices did indeed exhibit a large fractional change over astrophysically interesting scales, so that a powerlaw description of the scale dependence of the metric perturbations is not strictly valid. If 
the absolute value of the change is much less than unity, then the fact that $\Delta n_{T}>n_{T}$ or $\Delta(n-1)>(n-1)$ is undetectable and of little practical significance, and, as we shall show now, reconstruction can proceed. We refer to this as "weak reconstructability," or WR. A case in point is the natural inflation model [16]; with parameters chosen to give $(n-1)=-0.3$, the tensor spectral index grows by a factor of about 100 between the largest and smallest interesting scales. However, this growth is entirely unobservable, being the difference between $n_{T}=-10^{-9}$ and $n_{T}=-10^{-7}$.

Logically, there are three cases of WR: (i) scalar index satisfies SR and tensor index satisfies WR; (ii) tensor index satisfies SR and scalar index satisfies WR; and (iii) both tensor and scalar indices satisfy WR. Since we have previously derived the bounds to $m_{\mathrm{Pl}}^{m} V^{(m)} / V$ that follow if tensor and scalar indices satisfy SR, cf. Eqs. (28) and (29) respectively, here we simply do the same for WR. In case (i) the SR scalar and WR tensor bounds apply; in case (ii) the WR scalar and SR tensor bounds apply; and in case (iii) the scalar and tensor WR bounds apply. In all three cases the implications for convergence of the power series and the ordering of terms is very similar to the case of SR.

Let us take $\delta$ to be the parameter that quantifies the smallness of the tensor (or scalar) index and its absolute change. For sake of definiteness, we would imagine that a change of a few hundredths for the scalars, and considerably more for the tensors, would be extremely hard to observe. Following the same strategy as in the SR case, this time bounding the absolute change in the spectral index due to higher-order derivatives, we find:

$$
\begin{aligned}
& \left(\frac{m_{\mathrm{Pl}}^{m} V^{(m)}}{V}\right)<n_{T}^{-m / 2} \Delta N^{-m+1} \delta(m-1) ! \quad \text { for } m \geq 2 \text { (tensor) } \\
& \left(\frac{m_{\mathrm{Pl}}^{m} V^{(m)}}{V}\right)<n_{T}^{(1-m) / 2} \Delta N^{-m+2} \delta(m-2) ! \quad \text { for } m \geq 3 \text { (scalar). }
\end{aligned}
$$

These constraints differ from their counterparts in the SR case only slightly: by one fewer factor of $n_{T}$ (tensor) and by the absence of the $(n-1)$ factor (scalar). Thus, the conclusions reached for convergence and term ordering in the SR case carry over with only minor modification. For example, the size of the contribution of the $m$-th derivative to the Taylor series of the potential is bounded by $(n-1) n_{T} \Delta N^{2} \delta / m(m-1), n_{T} \Delta N^{2} \delta / m(m-1)$, and $n_{T} \Delta N^{2} \delta / m(m-1)$ in cases (i)-(iii) respectively; this guarantees absolute convergence. If the SR tensor bound applies then a term involving $m$ derivatives is as before bounded by $n_{T} \delta / \Delta N^{m / 2-1}$; if the WR tensor bound applies then such a term is bounded by $\delta / \Delta N^{m / 2-1}$.

Finally, what types of potentials give rise to order unity fractional changes in the spectral indices while still satisfying the WR criteria? It is simple to show for the tensor index that $\Delta N(n-1)$ must be of order unity or larger; this occurs in the previously mentioned natural inflation model. For the scalar index the condition is that $\left(m_{\mathrm{Pl}}^{3} V^{(m)} / V\right) n_{T} \Delta N /(n-1)$ must be of order unity or larger. 


\subsection{Sensibility summary}

When physicists construct an expansion in a small parameter (or even several small parameters) they rarely worry about rigorous mathematical issues. While we would like to follow in that tradition, the problem here is a bit more vexing as there are in principle an infinity of small expansion parameters: $m_{\mathrm{Pl}}^{m} V^{(m)} / V$. We have addressed two (not unrelated) issues here: convergence of the reconstructed potential and ordering of terms.

Based upon pragmatic criteria that derive from the data themselves we have shown that convergence and term ordering follow for potentials where the spectral indices do not vary significantly over astrophysically interesting scales (referred to as SR), or if they do vary by of order unity, the absolute change is small by comparison to what can be measured (referred to as WR). In both cases we explicitly showed that the Taylor expansion for the potential is necessarily convergent, and that higher-derivative terms in the expansions for the observables descend in size. For "scale-free" potentials a term that involves $m$ derivatives is of the order

of $n_{T}^{m / 2}$; in the more generic cases of SR and WR, such a term is bounded by $n_{T} \delta / \Delta N^{m / 2-1}$ and $\delta / \Delta N^{m / 2-1}$ respectively. This establishes what has been previously assumed implicitly in Refs. [9, 13]: the terms involving more derivatives are of higher order.

\subsection{The consistency relation}

An important feature of reconstruction is that the problem is overdetermined; specifically, a set of $M \geq 3$ observables can be expressed in terms of the potential and its first $M-2$ derivatives. This implies a "consistency relation," which, for increasing $M$, contains terms of higher and higher order. The lowest-order consistency equation, $n_{T}=-\frac{1}{7} \frac{T}{S}$, has been much discussed (e.g., in Ref. [4, 5]) and arises through Eqs. (四), (8) and (国) which express $n_{T}, S$, and $T$ in terms of $V_{50}$ and $V_{50}^{\prime}$.

Calculating higher derivatives alone, while keeping the calculation of each derivative to lowest order, does not lead to the correct second-order term in the consistency equation, and nor does calculating the second-order corrections to the derivatives present. One must systematically do both. The second-order version of the consistency equation is obtained by calculating the potential, its derivative and Eq. (何) to a higher order. Adding an extra order to the calculation of $V_{50}^{\prime}$ adds a new observable, $(n-1)$, which will appear in the consistency equation at second order. To account for there being still only a single consistency equation, there must be a new equation, and because $(n-1)$ has only entered at second-order in $V_{50}^{\prime}$, we only need the first-order equation for $V_{50}^{\prime \prime}$. The second-order consistency equation, which we calculate in this paper, therefore relates $n_{T}, \frac{T}{S}$ and $(n-1)$, with the last only appearing as a second-order correction. Were one to desire a calculation to yet higher order, the same pattern would persist; each existing derivative must be calculated to one extra order and the next derivative to lowest order, introducing a new observable. This will generate nextorder terms in the consistency equation with the new observable appearing at that order. However, this presently cannot be done as third-order expressions for $V_{50}$ and $V_{50}^{\prime}$ have not been calculated. 


\subsection{Expansion techniques}

Given the value of the potential and its first two or three derivatives at a point and the $\phi_{N}$ relation just obtained, one can reconstruct the potential on the observationally relevant scales (i.e., $N \simeq 42-50$ ). The standard technique used previously is the Taylor expansion

$$
V(\phi)=V_{50}+V_{50}^{\prime}\left(\phi-\phi_{50}\right)+\frac{1}{2} V_{50}^{\prime \prime}\left(\phi-\phi_{50}\right)^{2}+\cdots
$$

For many situations this is perfectly fine (e.g., when $n_{T}$ and $n-1$ are small, see Ref. [12]). However, if the range of eight or so e-foldings corresponds to a large range in $\phi$ the convergence may not be very good because of the abrupt truncation of the Taylor series. Specifically, for large $\left(\phi-\phi_{50}\right)$ the shape of the reconstructed potential is dictated, rightly or wrongly, by the last term in the expansion (quadratic or cubic).

An alternative is the Padé approximant [17], which can be generated directly from a truncated power series. For a power series that extends to order $N$, the Padé approximants are quotients of two polynomials of order $L$ (numerator) and $M$ (denominator) denoted by $[L, M]$, where $L+M=N$. By construction, the expansion of $[L, M]$ matches that of the power series to order $N$, but of course is not truncated. Very often, Padé approximants provide a very good approximation over a wider range of values than the Taylor series from which they are derived; they in some way encode better estimates of the higher-order terms than does truncation. If we truncate the Taylor series at the second derivative, then the associated diagonal Padé approximant $[1,1]$ is a ratio of two first-order polynomials given by包

$$
R(\phi)=\frac{a_{0}+a_{1}\left(\phi-\phi_{50}\right)}{1+b_{1}\left(\phi-\phi_{50}\right)}
$$

with

$$
a_{0}=V_{50} ; \quad b_{1}=-V_{50}^{\prime \prime} / 2 V_{50}^{\prime} ; \quad a_{1}=V_{50}^{\prime}-V_{50} V_{50}^{\prime \prime} / 2 V_{50}^{\prime} .
$$

As we shall illustrate later by specific example, Padé approximants have a lot to offer when the Taylor series proves a poor approximation.

\section{Second-order Reconstruction Reduced to Practice}

Having discussed the philosophy and strategy, let us proceed to deriving the full reconstruction equations at second-order. The reconstruction equations for the scalar potential and its first two derivatives, evaluated to second-order, are given in Ref. [13], though not in terms of cosmological observables. They are given in terms of the perturbation amplitudes $A_{G}^{2}$ and $A_{S}^{2}$. Very roughly, $A_{S}$ is the horizon-crossing amplitude of the density perturbation on a given scale and $A_{G}$ is the horizon-crossing amplitude of the tensor perturbation (in the

\footnotetext{
${ }^{5}$ The $[2,0]$ approximant is just the truncated Taylor series; in addition to simplicity, there is some motivation for using the diagonal approximant rather than the $[0,2]$ approximant as it is asymptotically constant, consistent with the flatness of inflationary potentials.
} 
Appendix we provide some relations between notation used in that paper and this one.) Our purpose here is to express these second-order expressions for the potential and its first two derivatives in terms of the measurable quantities $n, d n / d \ln k, n_{T}, S$, and $T$.

The amplitudes $A_{S}^{2}$ and $A_{G}^{2}$ are related to the observables $S, T, n_{T}$ and $n$ by:

$$
A_{G}^{2}=0.70\left(1-1.3 n_{T}\right) T, \quad A_{S}^{2}=9.6[1-1.15(n-1)] S,
$$

where the $(n-1)$ and $n_{T}$ dependencies have been found by evaluating the Sachs-Wolfe integrals numerically. Both expressions are accurate to second-order.

Before deriving second-order expressions for the potential and its derivatives, we calculate the second-order version of the consistency relation. It is obtained from Eq. (2.9) of Ref. [13],

$$
-\frac{n_{T}}{2}=\frac{A_{G}^{2}}{A_{S}^{2}}[1+3 \epsilon-2 \eta],
$$

where to the required order the slow-roll parameters $\epsilon$ and $\eta$ (defined in the Appendix) are given by

$$
\epsilon=-n_{T} / 2, \quad \eta=(n-1) / 2-n_{T} .
$$

This gives a simple and very useful relation for $A_{G}^{2} / A_{S}^{2}$,

$$
\frac{A_{G}^{2}}{A_{S}^{2}}=-0.5 n_{T}\left[1-0.5 n_{T}+1.0(n-1)\right] .
$$

Substituting into Eq. (36), we find the second-order consistency relation

$$
n_{T}=-\frac{1}{7} \frac{T}{S}\left[1-0.8 n_{T}+0.15(n-1)\right],
$$

or

$$
\frac{T}{S}=-7 n_{T}\left[1+0.8 n_{T}-0.15(n-1)\right]
$$

To the required order we can use the first-order truncation $n_{T}=-\frac{1}{7} \frac{T}{S}$ inside the brackets, thereby obtaining an alternative form,

$$
n_{T}=-\frac{1}{7} \frac{T}{S}\left[1+0.11 \frac{T}{S}+0.15(n-1)\right]
$$

where $n_{T}$ is given in terms of the more accessible quantities $(n-1)$ and $\frac{T}{S}$.

Independent measurements of $n, n_{T}$ and $\frac{T}{S}$ provide a powerful test of the inflationary hypothesis; in the space of these parameters inflationary models must lie on the surface defined by Eq. (41). In Figure 1 we illustrate the inflationary surface both without and with second-order corrections. The second-order corrections break the degeneracy in the $(n-1)$ direction, as well as typically reducing $\frac{T}{S}$ viewed as a function of $n_{T}$ and $(n-1)$. However, the portions of the surface that feature large corrections are not favored by present cosmological data, and further, are susceptible to higher-order corrections. (Indeed, well 
away from scale-invariance the surface would be noticeably different even just using Eq. (42) instead of Eq. (41), which differ by third and higher order terms.)

Obtaining the reconstruction equations is simply a matter of substituting into Eqs. (3.4), (3.6) and (3.15) of Ref. [13] for $V, V^{\prime}$ and $V^{\prime \prime}$ respectively. We give two alternative forms for each, the first using $n_{T}$ and the second substituting $\frac{T}{S}$ for $n_{T}$ using the second-order consistency equation. They are

$$
\begin{aligned}
V_{50} / m_{\mathrm{Pl}}^{4}= & 1.65\left(1-1.4 n_{T}\right) T, \\
= & 1.65\left(1+0.20 \frac{T}{S}\right) T, \\
V_{50}^{\prime} / m_{\mathrm{Pl}}^{3}= & \pm 8.3 \sqrt{-n_{T}}\left[1-1.1 n_{T}-0.03(n-1)\right] T, \\
= & \pm 8.3 \sqrt{\frac{1}{7} \frac{T}{S}}\left[1+0.21 \frac{T}{S}-0.04(n-1)\right] T, \\
V_{50}^{\prime \prime} / m_{\mathrm{Pl}}^{2}= & 21\left[(n-1)-3 n_{T}+1.4 n_{T}^{2}\right. \\
& \left.+0.6 n_{T}(n-1)-0.2(n-1)^{2}+1.1 \frac{d n}{d \ln k}\right] T, \\
& 21\left[(n-1)+0.43 \frac{T}{S}+0.073\left(\frac{T}{S}\right)^{2}\right. \\
& \left.-0.015 \frac{T}{S}(n-1)-0.2(n-1)^{2}+1.1 \frac{d n}{d \ln k}\right] T .
\end{aligned}
$$

These expressions are accurate to second-order. Naturally, they agree with the first-order expressions given earlier.

Though no expression is given in Ref. [13 for $V^{\prime \prime \prime}$, by using the lowest-order expressions for $\epsilon, \eta$, and a third slow-roll parameter $\xi$, and Eq. (3.13) which relates the three to $d n / d \ln k$, one can obtain the first-order expression,

$$
\begin{aligned}
V_{50}^{\prime \prime \prime} / m_{\mathrm{Pl}} & = \pm 104 \sqrt{-n_{T}}\left[\frac{d n / d \ln k}{n_{T}}-6 n_{T}+4(n-1)\right] T \\
& = \pm 104 \sqrt{\frac{1}{7} \frac{T}{S}}\left[-7 \frac{d n / d \ln k}{T / S}+0.9 \frac{T}{S}+4(n-1)\right] T .
\end{aligned}
$$

where the overall sign is to be the same as that of $V^{\prime}$. The second-order term would require yet another observable. As remarked in Ref. [13], even this first-order expression features the rate of change of the scalar spectral index, which is likely to be very difficult to measure. Realistically then, in the near term only the value of the potential and its first two derivatives are likely to be accessible to accurate determination.

The final step in reconstructing the potential is to use $d \phi / d N$ to the desired order, to find the range of $\phi$ that corresponds to the eight or so e-foldings of inflation relevant for astrophysics. To proceed, we may simply carry out a Taylor expansion of $\phi$ about $\phi_{50}$, to 
whatever order we believe is appropriate,

$$
\phi_{N}-\phi_{50}=\left.(N-50) \frac{d \phi}{d N}\right|_{\phi_{50}}+\left.\frac{1}{2}(N-50)^{2} \frac{d^{2} \phi}{d N^{2}}\right|_{\phi_{50}}+\cdots
$$

This is a double expansion, in the sense that the coefficients are themselves obtained as a series expansion in the slow-roll parameters.

To proceed, we use as a starting point the exact formula

$$
\dot{\phi}=-\frac{m_{\mathrm{Pl}}^{2}}{4 \pi} H^{\prime}
$$

which, along with $d N / d t=-H$, yields the relation from which the Taylor coefficients may be calculated,

$$
\frac{d \phi}{d N}=\frac{m_{\mathrm{Pl}}^{2}}{4 \pi} \frac{H^{\prime}}{H}
$$

To get a given coefficient in the Taylor expansion for $\phi_{N}$, one simply calculates $d^{i} \phi / d N^{i}$ expanding to the desired order in the deviation from scale invariance. Đ For example, taking only the first term in the $\phi_{N}$ expansion and working to first-order yields the expression already given in Section 1.1. We give the first coefficient in the $\phi_{N}$ expansion to secondorder and the second coefficient in the $\phi_{N}$ expansion to first-order only,

$$
\begin{aligned}
\phi_{N}-\phi_{50}= & \pm \frac{m_{\mathrm{Pl}}}{\sqrt{8 \pi}} \sqrt{-n_{T}}\left[1+0.1 n_{T}+0.1(n-1)\right](N-50) \\
& \pm \frac{m_{\mathrm{Pl}}}{4 \sqrt{8 \pi}} \sqrt{-n_{T}}\left[(n-1)-n_{T}\right](N-50)^{2}+\cdots
\end{aligned}
$$

with both signs again agreeing with that of $V^{\prime}$.

In the process of reconstruction, we shall use the first-order expansion for $\phi_{N}-\phi_{50}$ in first-order reconstruction, and the second-order expansion in second-order reconstruction.

\section{Reconstructing an exponential potential}

A useful testing ground for reconstruction is the exponential potential, the only known case where the perturbation spectra can be derived exactly analytically [9, 18]. For the potential

$$
V(\phi)=V_{0} \exp \left(-\sqrt{\frac{16 \pi}{p}} \frac{\phi}{m_{P l}}\right),
$$

\footnotetext{
${ }^{6}$ Note this procedure differs slightly from that in Ref. [12], where $d \phi / d N$ was expanded linearly about $\phi_{50}$ and $\phi_{N}$ was solved for exactly, cf. Eq. (8). This results in an exponential, whose expansion picks up the $(N-50)$ and $(N-50)^{2}$ terms correctly to lowest order in the deviation from scale invariance, though not the higher-order terms in the $(N-50)$ term which would require higher-order terms in the expansion of $d \phi / d N$. There is an overall sign error in Eq. (8) of Ref. [12].
} 
the scale factor grows exactly as $t^{p}$. Compared with the lowest order expressions, the amplitudes $A$ and $A_{T}$, or $A_{S}^{2}$ and $A_{G}^{2}$, are both multiplied by the same $p$-dependent factor $R^{2}(p)$, where

$$
R(p)=2^{1 /(p-1)} \frac{\Gamma[3 / 2+1 /(p-1)]}{\Gamma[3 / 2]}(1-1 / p)^{p /(p-1)},
$$

where $\Gamma(\cdots)$ is the usual gamma function. Both scalar and tensor spectra are exact power laws with spectral indices $(n-1)=n_{T}=-2 /(p-1)$. The scalar-field solution is characterized by

$$
\dot{\phi}=\sqrt{\frac{p}{4 \pi}} \frac{m_{\mathrm{Pl}}}{t} ; \quad \frac{d \phi}{d N}=-\frac{m_{\mathrm{Pl}}}{\sqrt{4 \pi p}} ; \quad V\left(\phi_{N}\right)=V_{50}^{\text {true }} \exp [2(N-50) / p] .
$$

The expressions for $T$ and $S$ can be obtained exactly by integrating Eqs. (5) and (6),

$$
\begin{aligned}
S & =2.2 f(n) R^{2}(p) \frac{V_{50}^{\text {true }}}{m_{\mathrm{Pl}}^{4} x_{50}^{2}} \\
T & =0.61 g\left(n_{T}\right) R^{2}(p) \frac{V_{50}^{\text {true }}}{m_{\mathrm{Pl}}^{4}},
\end{aligned}
$$

where the numerical factors $f(n)=1+1.15(n-1)+\cdots$ and $g\left(n_{T}\right)=1+1.3 n_{T}+\cdots$ arise from the $n, n_{T}$ dependence of the Sachs-Wolfe integrals, cf. Eqs. (5, 6).

We are now ready to carry out an array of reconstruction methods. Because we are using exact expressions to generate the spectra, this procedure is more ambitious, and more realistic, than those attempted thus far [6, 12], where the trial spectra were produced using the slow-roll approximation. For the general inflationary potential, exact results are not known, and so this procedure is not possible'. However, our method here should give a more realistic estimate of inherent errors even in the general case.

There are two distinct types of error. The first is error in the value of the potential at $\phi_{50}$, due to third-order and higher terms. By substituting the expression for $T$ in Eq. (55) into Eq. (10) or (43) for $V_{50}$ we can compute that error:

$$
V_{50} / V_{50}^{\text {true }}=g\left(n_{T}\right)\left(1-1.4 n_{T}\right) R(p)^{2} .
$$

The second error involves the shape of the potential, which depends on the ability of the chosen expansion to match the potential over the eight interesting e-foldings.

We have chosen as a specific example an exponential potential with $p=43 / 3$. We did so because this leads to about the largest departure from scale invariance that can still be regarded as observationally viable, $(n-1)=n_{T}=-0.15$ and $\frac{T}{S} \simeq 1$, and thus realistically represents the most challenging example of reconstruction. The exact potential is shown in Fig. 2 along with the results of five different reconstructions.

To begin, consider the error in estimating $V_{50}^{\text {true }}$; we have $g\left(n_{T}=-0.15\right)=0.824$ and so $V_{50} / V_{50}^{\text {true }} \simeq 0.95$, a modest $5 \%$ error due to the neglected higher-order terms. As we always

\footnotetext{
${ }^{7}$ Of course, we are going to pretend that we don't know the potential is exponential to demonstrate our methods.
} 
include the second-order term in $V_{50}$, the error is the same in every method we look at. Had the first-order expression for $V_{50}$ been used instead, corresponding to the neglect of the factor of $\left(1-1.4 n_{T}\right)$ in Eq. (10), then the underestimation would have been about $20 \%$.

Let us now consider the shape, which we note depends on $T$ and $S$ only through their ratio. The important distinction between different methods is the difference in required input data; methods needing only $n$ and $\frac{T}{S}$ have the advantage of depending only on the information that is easiest to obtain. Requiring $d n / d \ln k$ in addition, while offering more accuracy, is setting a much trickier observational task, though upper bounds are also useful in the absence of actual determinations.

As a starting point, let us take the equations derived in Ref. [12], which are primarily first-order though they include the second-order correction to $V_{50}$, cf. Eqs. (10 12). In this extreme example, the quadratic Taylor series based upon this does a bad job of approximating the shape of the potential, as it turns upward for large $\left(\phi-\phi_{50}\right)$ due to the truncation at the $\left(\phi-\phi_{50}\right)^{2}$ term (see Fig. 2).

If we now require knowledge of $d n / d \ln k$, the Taylor series approach can be improved in two ways. We can now take $V_{50}, V_{50}^{\prime}$, and $V_{50}^{\prime \prime}$ to second-order; however, the improvement is rather minimal. Alternatively, we can stick to first-order expressions, but include the $V_{50}^{\prime \prime \prime}$ cubic term. Again the improvement is modest, though at least the unwanted minimum has been eliminated. One could go further and take $V_{50}, V_{50}^{\prime}$, and $V_{50}^{\prime \prime}$ to second-order and $V_{50}^{\prime \prime \prime}$ to lowest order, which we haven't illustrated, again seeing only modest gains for the increased observational requirement.

The Taylor series having been unimpressive, let us progress in a different direction. With only $n$ and $\frac{T}{S}$, as an alternative to the Taylor series one can construct the Padé approximant based upon it, taking $V_{50}$ to second-order and $V_{50}^{\prime}$ and $V_{50}^{\prime \prime}$ to first-order. This represents a substantial gain on the Taylor series to that order without requiring any additional input information. With this minimal information, it is a much better method. Reintroducing $d n / d \ln k$ allows this method to be extended to second-order, where the reproduction of the shape of the potential is excellent. To include the third derivative term would necessitate a more complicated (non-diagonal) Padé approximant, which doesn't seem warranted at the moment.

What is the upshot of this comparison? Recalling that we have chosen an example with extreme deviation from scale-invariance, the second-order corrections are reassuringly small and only improve the shape of the reconstructed potential slightly. The addition of the third derivative term in the Taylor series gives a slightly more significant improvement, but at the price of its dependence upon $d n / d \ln k$ even at lowest order. The most remarkable improvement involves the use of Padé approximants. Even without knowledge of $d n / d \ln k$ the shape of the potential is reproduced far better than with the higher-order Taylor series which does require that knowledge. As noted previously, the improvement results from the fact that the Padé approximant is not truncated; further, even in situations where truncation of the Taylor series does not lead to problems, the Padé approximant still proves valuable as its Taylor expansion coincides with that of the original expansion. We therefore conclude that Padé approximants provide a significant improvement in the perturbative reconstruction 
of the inflationary potential.

\section{Discussion}

By presenting the second-order reconstruction equations directly in terms of observables, we have been able to assemble and to compare an array of different perturbative reconstruction techniques based upon cosmological observables. Our work extends previous work in several important ways.

First, we have placed the perturbative reconstruction process on a firmer foundation by addressing the important issues of convergence and term ordering. We have emphasized that the observational data themselves can be used to decide whether or not perturbative reconstruction is well justified and sensible. In particular, we have shown that the Taylor series for the potential is absolutely convergent and that terms in the expansions for the observables must decrease in size as the number of derivatives increase for the case where the spectral indices do not vary significantly over the astrophysically interesting scales, or, if they do, their absolute change is small.

Perhaps our most interesting result is the introduction of the Padé approximant as an alternative to the Taylor series in perturbative reconstruction. It can be obtained from a Taylor series regardless of the order (in the deviation from scale invariance) to which the coefficients of the Taylor series has been obtained. In our worked example, the improvement in reproducing the shape of the potential as compared to the Taylor series is striking, especially considering that no extra observables are required.

We have shown that the second-order corrections to the Taylor series coefficients are generally small, and that those for $V_{50}$ and $V_{50}^{\prime}$ only depend upon the same quantities as the first-order expressions $(S, T$, and $n)$. The corrections to $V_{50}^{\prime \prime}$ however require a new observable such as $d n / d \ln k$, and by deriving for the first time an explicit expression we have confirmed that even the lowest-order term in $V_{50}^{\prime \prime \prime}$ requires this challenging observable.

Finally, one of the most important aspects of reconstruction is that it is overdetermined: Any set of cosmological observables supplies degenerate information regarding the potential and its derivatives, thereby providing an important consistency check. In particular, the tensor spectral index can be expressed to second-order in terms of $S, T$, and $n$ by the

relation: $n_{T}=-\frac{1}{7} \frac{T}{S}\left[1+0.11 \frac{T}{S}+0.15(n-1)\right]$. In cases that are observationally viable, the second-order corrections are small.

\section{Acknowledgements}

We are grateful to John Barrow for bringing Padé approximants to our attention and to Sharon Vadas for many stimulating discussions. We also thank Ed Copeland, Scott Dodelson, Rocky Kolb, and Jim Lidsey. ARL was supported by the Royal Society, and acknowledges the hospitality of Fermilab where this work was initiated and also the use of the Starlink computer system at the University of Sussex. MST was supported in part by the Department 
of Energy (at Chicago and Fermilab) and by the NASA through grant NAGW-2381 (at Fermilab). ARL and MST both acknowledge the hospitality of the CfPA, Berkeley, while some of this work was carried out.

\section{Appendix: Some Relations between Notation}

For the convenience of the reader, we summarize here some relations between the notation used here and that in Ref. [13], from which several important results were taken. In that paper, the spectra $A_{S}$ and $A_{G}$ were defined so as to include any scale-dependence within them, i.e., they are functions of $k$. In circumstances where the spectra can be approximated by power-laws, these are related to the amplitudes $A$ and $A_{T}$ in this paper, which are just numbers, by

$$
\begin{aligned}
A\left(k / k_{50}\right)^{n-1} & =\frac{2 \pi^{2}}{H_{0}^{4}} A_{S}^{2}(k), \\
A_{T}\left(k / k_{50}\right)^{n_{T}} & =2 A_{G}^{2}(k) .
\end{aligned}
$$

Even in cases where the spectra cannot be described by power-laws, the correspondence holds at $k=k_{50}$.

In Ref. [13, slow-roll parameters $\epsilon$ and $\eta$ are introduced,

$$
\epsilon=\frac{m_{P l}^{2}}{4 \pi}\left(\frac{H^{\prime}}{H}\right)^{2}, \quad \eta=\frac{m_{P l}^{2}}{4 \pi} \frac{H^{\prime \prime}}{H},
$$

which are again in general $k$-dependent. As indicated in Section 2 of the present paper, they can be related to the spectral indices to various orders, $\epsilon$ and $\eta$ being of the same order in perturbation theory as $(n-1)$ and $n_{T}$. To lowest-order they are constant, corresponding to power-law spectra. At lowest-order $\epsilon=16 \pi x^{2}$, but higher order corrections break this relation.

\section{References}

[1] See e.g., E. W. Kolb and M. S. Turner, The Early Universe (Addison-Wesley, Redwood City, CA, 1990), Ch. 8.

[2] A. H. Guth and S.-Y. Pi, Phys. Rev. Lett. 49, 1110 (1982); A. A. Starobinskii, Phys. Lett. B117, 175 (1982); S. W. Hawking, ibid B115, 295 (1982); J. M. Bardeen, P. J. Steinhardt, and M. S. Turner, Phys. Rev. D28, 679 (1983).

[3] V. A. Rubakov, M. Sazhin, and A. Veryaskin, Phys. Lett. B115, 189 (1982); R. Fabbri and M. Pollock, ibid B125, 445 (1983); L. Abbott and M. Wise, Nucl. Phys. B244, 541 (1984); B. Allen, Phys. Rev. D37, 2078 (1988); L. P. Grishchuk, Phys. Rev. Lett. 70, 2371 (1993) and references therein. 
[4] A. R. Liddle and D. H. Lyth, Phys. Lett. B291, 391 (1992).

[5] M. S. Turner, Phys. Rev. D48, 3502 (1993).

[6] E. J. Copeland, E. W. Kolb, A. R. Liddle and J. E. Lidsey, Phys. Rev. Lett. 71, 219 (1993); Phys. Rev. D48, 2529 (1993).

[7] See e.g., G. Efstathiou, in The Physics of the Early Universe, edited by J.A. Peacock, A.F. Heavens, and A. T. Davies (Adam Hilgar, Bristol, 1990).

[8] M. S. Turner, M. White, and J. E. Lidsey, Phys. Rev. D48, 4613 (1993).

[9] E. D. Stewart and D. H. Lyth, ibid B302, 171 (1993).

[10] R. Crittenden, J. R. Bond, R. L. Davis, G. Efstathiou and P. J. Steinhardt, Phys. Rev. Lett. 71, 324 (1993).

[11] S. Dodelson, L. Knox and E. W. Kolb, "Testing Inflation with the Cosmic Microwave Background" Fermilab preprint FERMILAB-Pub-94/053-A (1994).

[12] M. S. Turner, Phys. Rev. D48, 5539 (1993).

[13] E. J. Copeland, E. W. Kolb, A. R. Liddle and J. E. Lidsey, Phys. Rev. D49, 1840 (1994).

[14] E. W. Kolb and S. L. Vadas, "Relating Spectral Indices to Tensor and Scalar Amplitudes in Inflation", Fermilab preprint FERMILAB-Pub-94/046-A (1994).

[15] J. D. Barrow and A. R. Liddle, Phys. Rev. D47, R5129 (1993).

[16] K. Freese, J. A. Frieman and A. V. Olinto, Phys. Rev. Lett. 65, 3233 (1990).

[17] See e.g. G. A. Baker, Jr. and P. Graves-Morris, Padé Approximants (Vol. 13 of the Encyclopedia of Mathematics, edited by G.-C. Rota) (Addison-Wesley, Reading, MA, 1981), or W. H. Press, S. A. Teukolsky, W. T. Vetterling and B. P. Flannery, Numerical Recipes (2nd edition) (Cambridge University Press, Cambridge, 1993).

[18] D. H. Lyth and E. D. Stewart, Phys. Lett. B274, 168 (1992).

\section{Figure Captions}

Figure 1: The consistency plane for inflation in $n-n_{T}-\frac{T}{S}$ space, the flat surface being the lowest-order result and the curved one incorporating the second-order corrections, given by Eq. (41).

Figure 2: An array of different reconstructions of an exponential potential with $(n-1)=$ $n_{T}=-0.15(p=43 / 3)$. The longer dotted line indicates the exact potential. The three 
different line styles correspond to three different reconstruction strategies; solid is Taylor series truncated at $\left(\phi-\phi_{50}\right)^{2}$, dashed is Taylor series truncated at $\left(\phi-\phi_{50}\right)^{3}$ and dashdotted is the Padé approximant based on the former of these. The upper line of a given style uses coefficients to first-order in the deviation from scale invariance (save $V_{50}$, which is always second-order), while the lower, where plotted, is second-order in all coefficients. The length of the curves corresponds to eight e-foldings. 
This figure "fig1-1.png" is available in "png" format from: http://arxiv.org/ps/astro-ph/9402021v2 
This figure "fig2-1.png" is available in "png" format from: http://arxiv.org/ps/astro-ph/9402021v2 


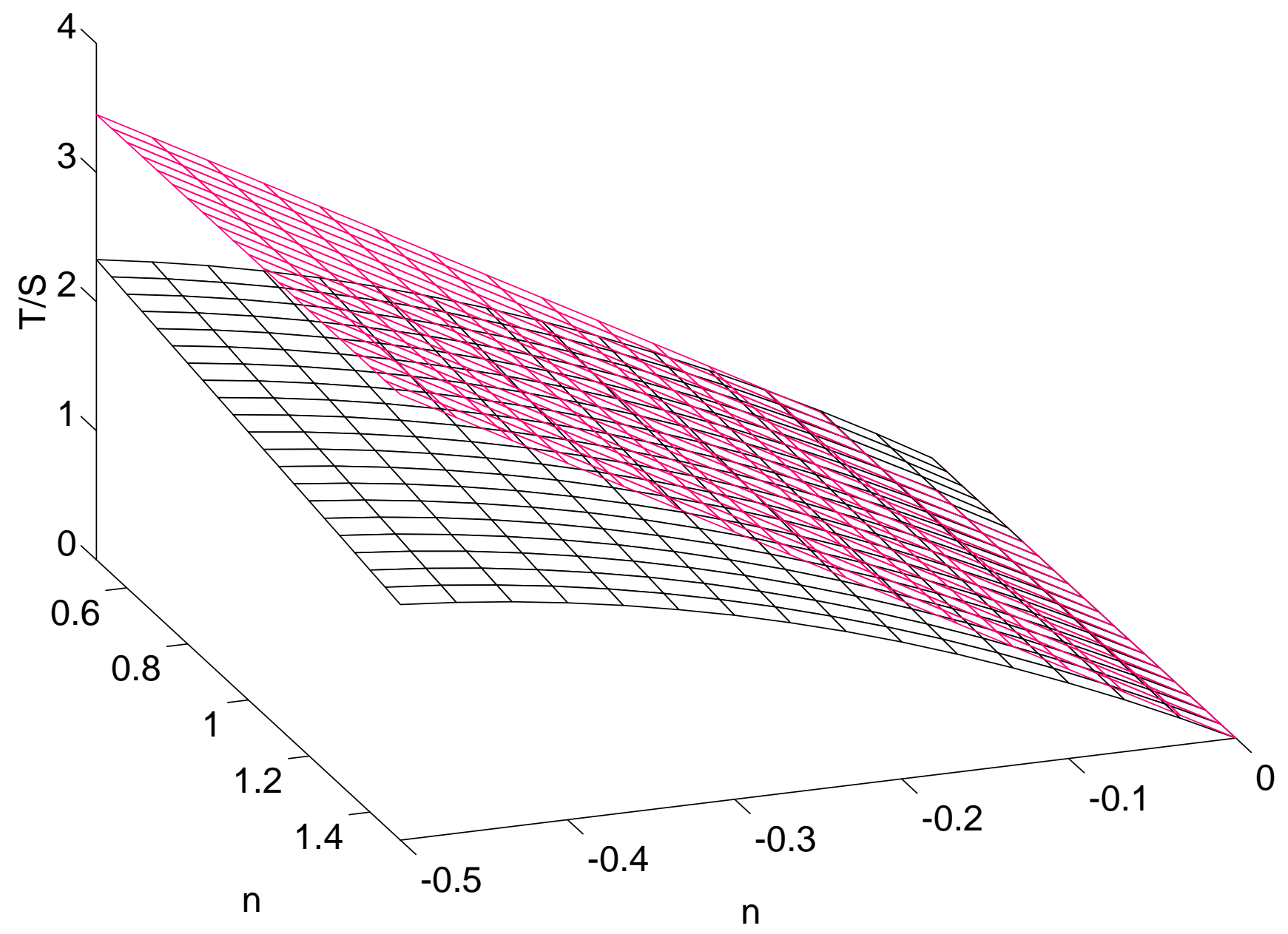




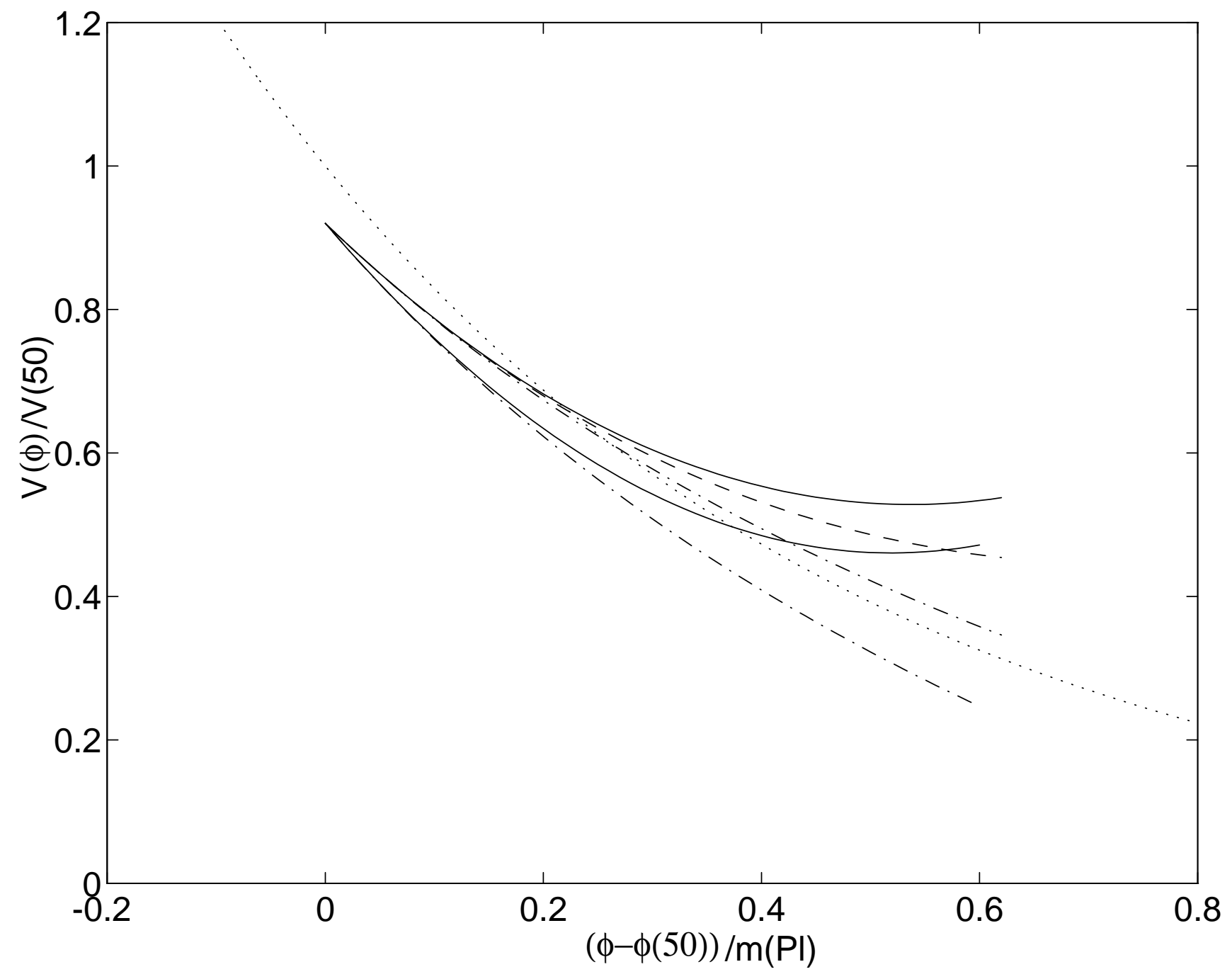

